\title{
Implementation of the Active TB Case Finding in Nigeria; Processes, Lessons Learnt and Recommendations
}

\author{
Oluremilekun Comfort Kusimo ${ }^{1 *}$, Richard Olukolade', Queen Ogbuji1, John Osho', \\ Adeyinka Onikan', Abiodun Hassan', Oladapo Alabi Ladipo', Oladipo Owoyomi², \\ Joseph Bakare ${ }^{2}$, Simeon Onyemaechi ${ }^{3}$, Adebola Lawanson ${ }^{3}$ \\ ${ }^{1}$ Programs Unit, Association for Reproductive and Family Health, Abuja, Nigeria \\ ${ }^{2}$ Programs Unit, Health Alive Foundation, Ilorin, Nigeria \\ ${ }^{3}$ Department of Public Health, National Tuberculosis and Leprosy Control Programme, Abuja, Nigeria \\ Email: *stremi2001@yahoo.com
}

How to cite this paper: Kusimo, O.C., Olukolade, R., Ogbuji, Q., Osho, J., Onikan, A., Hassan, A., Ladipo, O.A., Owoyomi, O., Bakare, J., Onyemaechi, S. and Lawanson, A. (2018) Implementation of the Active TB Case Finding in Nigeria; Processes, Lessons Learnt and Recommendations. Journal of Tuberculosis Research, 6, 10-18. https://doi.org/10.4236/jtr.2018.61002

Received: January 13, 2018

Accepted: March 4, 2018

Published: March 7, 2018

Copyright $\odot 2018$ by authors and Scientific Research Publishing Inc. This work is licensed under the Creative Commons Attribution International License (CC BY 4.0).

http://creativecommons.org/licenses/by/4.0/

\section{(c) (i) Open Access}

\begin{abstract}
Introduction: Tuberculosis remains a major public health issue in Nigeria with the country ranking seventh among the 30 high TB burden countries. Nigeria is among the three countries that account for half of the 4.3 million "missing" TB cases globally. One untreated TB case is able to infect about 10 20 people annually. Hence, it is pertinent that TB is controlled effectively through the use of active case finding strategies to find the missing TB cases. Objective: This article highlights the best practices and key lessons learnt during the implementation of the active house to house TB case finding strategy in Nigeria. Lessons Learnt and Recommendations: Strategic community engagement, massive awareness creation using mass media and local means of communication, mapping of locations where registered TB cases live, to inform home visits for contact tracing and strengthening of laboratory systems, are viable mechanisms to improve TB case finding. Conclusion: Active TB case finding interventions should be implemented in the context of a strengthened diagnostics system, strategic community engagement and media involvement.
\end{abstract}

\section{Keywords}

Active Case Finding, Tuberculosis, Lessons Learnt, Community Engagement

\section{Introduction}

Globally, tuberculosis (TB) remains a tremendous threat to human health [1]. 
TB is an infectious disease that is caused by Mycobacterium tuberculosis [2]. It is diagnosed using the Xpert ${ }^{\mathrm{R}} \mathrm{MTB} / \mathrm{RIF}$ assay or the sputum smear microscopy [2]. $\mathrm{TB}$ is prevalent throughout the globe and about 1.2 million lives are lost annually as a result of the disease [3]. Of these deaths, over $95 \%$ occur in low and middle-income countries which mostly have weak health systems and poorly funded national tuberculosis programmes (NTPs) [3]. Nigeria ranks seventh among the 30 high burden countries and is amongst the three countries that account for half of the 4.3 million "missing" TB cases globally [2] [4]. It is estimated that about $40 \%$ of incident TB cases each year are not notified [5]. Many of these cases are either never diagnosed or not documented. Undetected cases increase the risk of TB transmission as one untreated TB case is able to infect about 10 15 people annually [6] [7]. Hence, finding the missing cases is crucial to effective $\mathrm{TB}$ control and it is pertinent that TB is controlled effectively through case finding and treatment of active disease, treatment of latent $\mathrm{TB}$ infection and vaccination with Bacille Calmette-Guérin (BCG).

Despite the great strides achieved in the control of TB globally, the results of prevalence surveys and WHO estimates for many countries remain staggering, therefore the urgency to improve tuberculosis (TB) case finding remains one of the major concerns of national programs worldwide [5]. The WHO global TB control policy endorses the directly observed treatment short course (DOTS) strategy which relies on symptomatic individuals voluntarily seeking care at health facilities as the standard strategy for case finding [8]. Nigeria implements the DOTS strategy and has a treatment success rate of $84 \%$ for new and relapse cases including those notified from community interventions [2]. Passive self-presentation of patients has been shown to improve TB case detection in some high-TB burden countries such as Ethiopia, Peru and Vietnam, however the strategy may not be adequate in countries with high prevalence of HIV and suboptimal TB treatment coverage [7] [9] [10]. Consistent low TB case detection has been reported in Nigeria in spite of the huge investment in expansion of both diagnostics and treatment services in all parts of the country. In order to address this challenge, several innovative strategies were adopted to find the "missing TB cases". Key amongst them is the active house to house search for TB. This strategy was implemented in 20 states across six geopolitical zones of Nigeria through the support of the Global Fund for a period of two and a half years. This article seeks to highlight the best practices and key lessons learnt during the implementation of the active house to house strategy.

\section{Literature Review}

Active case finding interventions have been implemented in many high burden countries to reduce diagnostic delay and address low case finding [7] [9]. This approach has been shown to be effective in both clinical and community settings [11]. The strategy has been particularly successful in crowded urban populations in countries like Pakistan, Cape Town, Nepal, Kenya and South India, Nigeria among others [9] [12] [13] [14]. In many high burden countries, patients face 
barriers such as distance to the nearest health facility and lack of trained healthcare workers, to access TB services [15] [16]. These barriers have all been associated with patient and health system delays from the onset of illness to treatment initiation. The passive approach therefore becomes less effective in such settings because of limited access to TB services as a result of poor treatment coverage. Also, poor health seeking behavior has been identified as a barrier to accessing TB services and this is further escalated by stigma and poor attitude of health workers [17] [18] [19]. These limitations that are often found in low resource settings translate to the inadequacy of the passive case finding approaches to curb the spread of TB particularly in crowded urban populations commonly referred to as slums. There is ample evidence in the literature about the effectiveness of the various models of active case finding interventions such as house to house campaigns in slums, case finding among PLHIVs in the ART clinics and screening of clients in out-patient departments among others [7] [9]-[14]. There is however a gap in the documentation of the processes, challenges and the lessons learnt from the implementation of the various methods. Scale up of the active case finding strategies in high burden TB countries appears to be a step in the right direction, however scaling up of the strategies may also mean scaling up the weaknesses, especially if they have been left unsolved. Hence there is the need to better understand the strengths and weaknesses of the various active case finding models. Documentation of the lessons learnt and the best practices may increase the likelihood of success in countries with similar contexts and save resources that may be lost as a result of weak programmatic structures and processes.

This paper seeks to address this gap in the literature by providing a robust description of the process, lessons learnt and recommendations from the implementation of the active house to house case finding intervention in the urban crowded communities or "slums" in Nigeria.

\section{Geographical Coverage of Intervention}

The project engaged over 365 high risk communities from 20 states in the six geopolitical zones of Nigeria. These communities included the rural and urban crowded communities "slums" and few nomadic communities as shown in Figure 1.

\section{Processes for the Active Case Finding Intervention Include the Following}

1) The planning stage of the process involved a desk review to identify the areas with high burden of TB particularly in communities that are characterized by overcrowding and limited coverage of TB services. This was followed by a stakeholders meeting to discuss the operationalization of the strategy in the intervention areas as shown in Figure 2.

2) Community entry in the intervention areas entailed pre-advocacy visits to 


\section{COVERAGE OF ACTIVE CASE FINDING INTERVENTION IN NIGERIA}

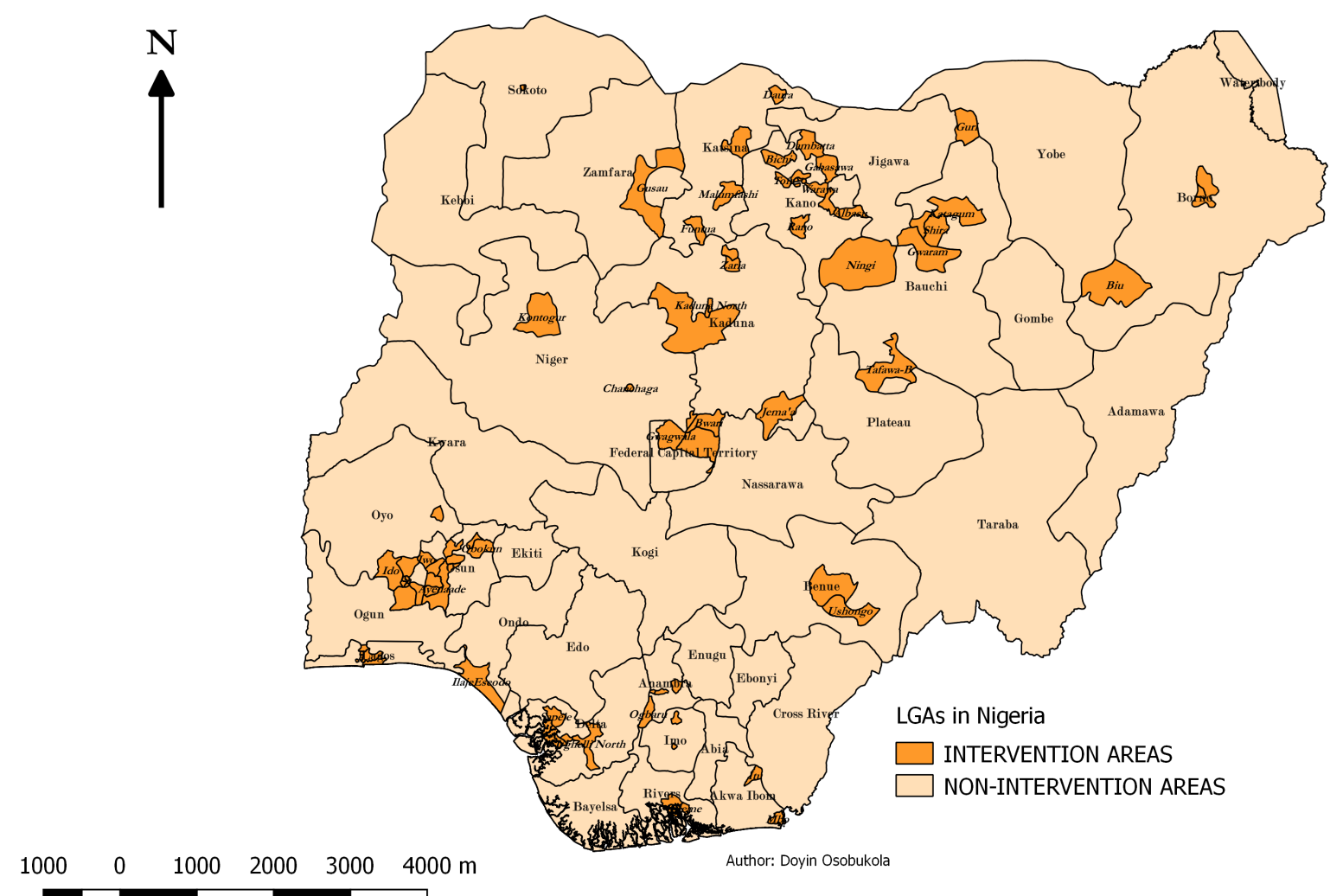

Figure 1. Geographical coverage of Global-Fund supported active case finding intervention in Nigeria.

Framework for the Implementation Process

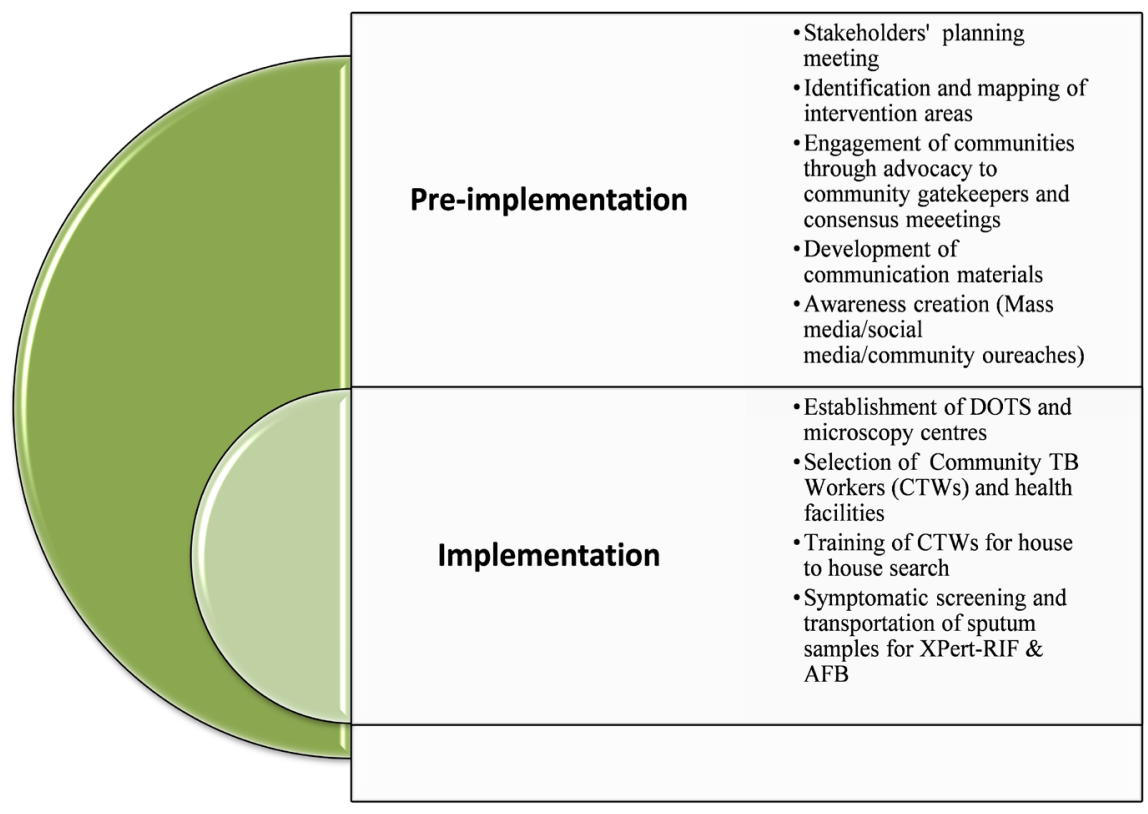

Figure 2. Framework for implementing the active case finding intervention. 
the states, local government areas (LGA) and slums to map the available health facilities including TB treatment and diagnostics services as well as key stakeholders that can drive the community engagement process. This was then followed by an advocacy visit to the identified key stakeholders (Ward Development Committee, Director of Primary Healthcare Agencies, religious/traditional leaders) in the community and an intensive awareness creation in the intervention areas using the mass media and community outreaches. Collection of baseline data was also done at this stage of the process. The baseline data was obtained from existing program records through the support of the State TB team and the LGA supervisor responsible for the intervention areas. During the community entry process, mapping of available community based organizations (CBOs), community volunteers and existing community structures that can support the implementation of the active case finding intervention was conducted.

2.1) Consensus Meeting/Community dialogues was held to define roles and responsibilities of the different stakeholders. This is to ensure that all the different stakeholders have a clear understanding of their roles and responsibilities. At this meeting, potential barriers to TB services and the challenges that may mitigate against the successful implementation of the intervention were identified. This activity is very important and should be held before the commencement of the roll-out of the active case finding intervention.

3) Selection of Community volunteers/community TB workers (CVs/CTWs) was conducted by the CBOs in collaboration with the community members, community gatekeepers, LGA TB supervisors and the State TB control Managers. Previous experience working on TB, malaria and HIV projects, being resident in the community, recognition in the community and ability to read and write were the key criteria for the selection of CVs/CTWs.

4) Training of $C B O s, C V s / C T W s$, Health Workers and TBLS for about two days on the processes involved in the active case search interventions \& how to ensure effective infection control procedures. This training was focused on the identification of presumptive TB cases, collection and transportation of sputum specimen, communication, and infection control as well as the data collection processes and tools for the intervention. The $\mathrm{CBOs}$ were also trained on how to conduct effective awareness about TB in the community and coordinate the activities of CTWs/CVs.

5) Awareness creation on $T B$ in the intervention areas for about two months before the implementation of the house to house visits for TB. This activity was implemented using mass media, Information, Education and Community (IEC) materials (in local languages) and local communication platforms such as town announcers.

6) House to house visits to identify presumptive TB cases by CTWs. The project entailed house to house visits by trained CTWs to identify presumptive TB cases, collect their sputum samples and transfer to GeneXpert centres or 
microscopy sites for screening. Consequently, those with positive laboratory test results were contacted and linked to treatment centers, while smear negative symptomatic cases were referred to the State TB Program for further investigation. Chronic cough ( $\geq 2$ weeks) inquiry was used as a screening tool. A CTW reached an average of 50 households weekly. It is important to note that one household in the slum can consist of an average of 20 - 40 or more persons and it usually takes several visits to meet with these persons because they are mostly out during the greater part of the day for economic reasons. CTWs also traced the contacts of registered $\mathrm{TB}$ patients during their house to house visits.

CBOs were responsible for oversight supervision of the activities of the CTWs and the coordination of the sputum movement to the TB diagnostic centres for testing. The $\mathrm{CBOs}$ were also expected to maintain communication with the facility workers and the trained CTWs. CTWs were remunerated monthly for the work done. The activities of the CTWs were monitored closely by CBOs, State TB team, National TB program and key implementing partners. The performance of the CTWs was reviewed periodically and poor performance resulted in withdrawal from the project. The benchmark for performance was defined as identifying presumptive TB cases from the allotted slum communities. To enhance performance, targets were set for CBOs and CTWs for the number of TB cases to find per quarter.

Sputum samples were collected by the CTWs from the identified presumptive TB case and were sent to the designated TB laboratory in the LGA. All bacteriologically positive or clinically diagnosed TB cases were treated in DOTS sites within the area. In line with the National Guidelines, all TB cases were screened for HIV at the DOTS centres and the HIV positive TB cases among them were linked to HIV services. The CBOs and CTWs ensured that patients placed on treatment completed their medication, however the CTWs may or may not be treatment supporters for a TB patient on treatment, since this is a prerogative of the patient.

\section{Lessons Learnt}

17,323 persons were notified in about a 24 -month period of implementation. This represents about $9 \%-12 \%$ of the total TB cases detected annually, in the country, between 2016 and 2017, hence, a key lesson learnt is that the house to house active $\mathrm{TB}$ case finding intervention is a viable approach to increase $\mathrm{TB}$ case finding in overcrowded communities. From the community perspective, lessons include the fact that strategic community engagement through consensus building meetings and dialogues is a crucial part of the active case finding intervention and can have significant impact on the outcome of the intervention. Intensive awareness creation using mass media and local means of communication is essential for community acceptance and ownership. Similarly, active involvement of community stakeholders in resource mobilization is essential for project sustainability. Many of the communities supported the renovation of laboratories, 
provision of water wells and alternative power supply to support the laboratory processes among others. Additionally, mapping of locations of registered TB cases as indicated in the treatment cards and facility registers to inform home visits, has the potential to increase the yield from the intervention. Also, a good working relationship between $\mathrm{CBO}$ and health workers is essential for a smooth implementation of intervention.

It is important to note that cultural and gender-related issues are a constant reality that cannot be ignored during the implementation of the intervention. In the northern part of the country, female visitors have access to households where women live with their families, while males' visitors are culturally prohibited from entering such households. Thus, in the affected northern locations, men who are CTWs, were restricted access into households where women were present. This cultural and gender-issues should be considered when recruiting the personnel that will be engaged in the house to house active TB case search intervention. A balanced mix of both male and female should be engaged and team work is highly recommended.

From the facility and systems perspective, motivation of health workers involved in the active case finding intervention through the provision of incentives is essential for achieving a successful outcome. Experience reveals that some staff at the diagnostic sites have to work overtime to cope with the increasing work load of testing sputum samples from the active TB case search intervention sites. Also, it is crucial that diagnostics capacity is strengthened in the intervention areas to optimize the yield of the intervention. Where there is inadequate coverage of Xpert ${ }^{R}$ MTB/RIF, establishment of diagnostics networks and support for sputum movement is recommended. Most importantly, supportive supervision and monitoring of the intervention by the major stakeholders, is a critical ingredient for the success of the intervention.

\section{Recommendations}

Active case finding interventions are costly and therefore should be implemented in the context of a strengthened diagnostics system for optimal yield. High negativity rate as a result of low sensitivity of diagnostic methods may result in poor return on investment in terms of the case yield and deaths averted based on the intervention. In line with this, expansion of diagnostic facilities (Xpert $^{\mathrm{R}} \mathrm{MTB} / \mathrm{RIF}$ ) in the intervention sites should be part of the preparatory processes. The diagnostics facilities should be subjected to regular potency and quality assurance measures, to ensure reliability of test results.

Strategic community and media involvement should be an integral part of the design of the intervention hence, adequate budgetary provision should be made for mass media engagement and the printing of IEC materials to reinforce awareness as well as generate demand for TB services within the communities. The creation of sensitization and awareness within the communities should inculcate a feedback mechanism from the people served with TB messages, 
through the provision of a dedicated toll free telephone line for people to solicit more information about TB services.

In line with increasing access to TB services, it is important to also regularly strengthen the capacity of the CBOs and CTWs to be able to identify presumptive TB cases through regular capacity building workshops. This should be budgeted and funded. It is equally important to continually provide these community actors with effective infection control and data management skills.

Community support and ownership of the intervention will no doubt produce impressive results. Thus, gatekeepers and other stakeholders in the communities should be involved actively in resource mobilization to ensure the sustainability of the project; especially in the light of the fact that donor funding is shrinking and government counterpart funding un-available in poor resource countries.

\section{References}

[1] Glaziou, P., Sismanidis, C., Floyd, K. and Raviglione, M. (2015) Global Epidemiology of Tuberculosis. Cold Spring Harbor Perspectives in Medicine, 5, 1-17. https://doi.org/10.1101/cshperspect.a017798

[2] WHO (2017) Global Tuberculosis Report 2017: Leave No One Behind-Unite to End TB.

[3] Bhutta, Z.A., Sommerfeld, J., Lassi, Z.S., Salam, R.A. and Das, J.K. (2014) Global Burden, Distribution, and Interventions for Infectious Diseases of Poverty. Infectious Diseases of Poverty, 3, 21. https://doi.org/10.1186/2049-9957-3-21

[4] WHO (2016) Global Tuberculosis Report 2016. World Health Organization, p. 211.

[5] Raviglione, M. and Sulis, G. (2016) Tuberculosis 2015: Burden, Challenges and Strategy for Control and Elimination. Infectious Disease Reports, 8, 33-37. https://doi.org/10.4081/idr.2016.6570

[6] Chigbu, L.N. and Iroegbu, C.U. (2010) Incidence and Spread of Mycobacterium tuberculosis-Associated Infection among Aba Federal Prison Inmates in Nigeria. Journal of Health, Population and Nutrition, 28, 327-332.

[7] Yuen, C.M., Amanullah, F., Dharmadhikari, A., Nardell, E.A., Seddon, J.A., Vasilyeva, I., et al. (2015) Turning off the Tap: Stopping Tuberculosis Transmission through Active Case-Finding and Prompt Effective Treatment. Lancet, 386, 2334-2343. https://doi.org/10.1016/S0140-6736(15)00322-0

[8] Murray, C.J.L. and Salomon, J.A. (1998) Expanding the WHO Tuberculosis Control Strategy: Rethinking the Role of Active Case-Finding [The Pittsfield Lecture]. International Journal of Tuberculosis and Lung Disease, 2, S9-S15.

[9] Mastinu, A., Stobbione, T., Rivetti, D., Oddone, M. and Terreno, M. (2016) Impact of Active TB Case Finding in a Population Resident in an Area of Northern Italy. Chest, 150, 279A. https://doi.org/10.1016/j.chest.2016.08.292

[10] Yimer, S., Holm-Hansen, C., Yimaldu, T. and Bjune, G. (2009) Evaluating an Active Case-Finding Strategy to Identify Smear-Positive Tuberculosis in Rural Ethiopia. International Journal of Tuberculosis and Lung Disease, 13, 1399-1404.

[11] Golub, J.E., Mohan, C.I., Comstock, G.W. and Chaisson, R.E. (2005) Active Case Finding of Tuberculosis: Historical Perspective and Future Prospects. International Journal of Tuberculosis and Lung Disease, 9, 1183-1203.

[12] Benson, U.O., Mobegi, D., Kiptai, T., Mueni, J., Mungai, M., Ngari, F., Mwamburi, 
C., Samoei, Z. and Kamau, C. (2016) Increasing Tuberculosis Yield from Investigation of Contacts of Smear Positive TB Cases through Engagement of Civil Society Organizations: Active TB Case Finding in Mombasa, Kenya. International Journal of Infectious Diseases, 45, 293-294. https://doi.org/10.1016/j.ijid.2016.02.647

[13] Ogbudebe, C.L., Chukwu, J.N., Nwafor, C.C., Meka, A.O., Ekeke, N., Madichie, N.O., et al. (2015) Reaching the Underserved: Active Tuberculosis Case Finding in Urban Slums in Southeastern Nigeria. International Journal of Mycobacteriology, 4, 18-24. https://doi.org/10.1016/j.ijmyco.2014.12.007

[14] Alemayehu, M., Gelaw, B., Abate, E., Wassie, L., Belyhun, Y., Bekele, S., et al. (2014) Active Tuberculosis Case Finding and Detection of Drug Resistance among HIV-Infected Patients: A Cross-Sectional Study in a TB Endemic area, Gondar, Northwest Ethiopia. International Journal of Mycobacteriology, 3, 132-138. https://doi.org/10.1016/j.ijmyco.2014.02.004

[15] Goudge, J., Gilson, L., Russell, S., Gumede, T. and Mills, A. (2009) Affordability, Availability and Acceptability Barriers to Health Care for the Chronically Ill: Longitudinal Case Studies from South Africa. BMC Health Services Research, 9, 75. https://doi.org/10.1186/1472-6963-9-75

[16] Tadesse, T., Demissie, M., Berhane, Y., Kebede, Y. and Abebe, M. (2013) Long Distance Travelling and Financial Burdens Discourage Tuberculosis DOTs Treatment Initiation and Compliance in Ethiopia: A Qualitative Study. BMC Public Health, 13, 424. https://doi.org/10.1186/1471-2458-13-424

[17] Li, Y., Ehiri, J., Tang, S.L., Li, D.K., Bian, Y.Q., Lin, H., Marshall, C. and Cao, J. (2013) Factors Associated with Patient, and Diagnostic Delays in Chinese TB Patients: A Systematic Review and Meta-Analysis. BMC Medicine, 11, 156. https://doi.org/10.1186/1741-7015-11-156

[18] Yang, W.-T., Gounder, C.R., Akande, T., De Neve, J.-W., McIntire, K.N., Chandrasekhar, A., de Lima Pereira, A., Gummadi, N., Samanta, S. and Gupta, A. (2014) Barriers and Delays in Tuberculosis Diagnosis and Treatment Services: Does Gender Matter? Tuberculosis Research and Treatment, 2014, Article ID: 461935. https://doi.org/10.1155/2014/461935

[19] Krishnan, L., Akande, T., Shankar, A.V., McIntire, K.N., Gounder, C.R., Gupta, A. and Yang, W.-T. (2014) Gender-Related Barriers and Delays in Accessing Tuberculosis Diagnostic and Treatment Services: A Systematic Review of Qualitative Studies. Tuberculosis Research and Treatment, 2014, Article ID: 215059.

https://doi.org/10.1155/2014/215059 